\title{
Random Extensions of Free Groups and Surface Groups are Hyperbolic
}

\section{Samuel J. Taylor and Giulio Tiozzo}

Department of Mathematics, Yale University, 10 Hillhouse Ave, New Haven, CT 06511, USA

Correspondence to be sent to: giulio.tiozzo@yale.edu

In this note, we prove that a random extension of either the free group $F_{N}$ of rank $N \geq 3$ or of the fundamental group of a closed, orientable surface $S_{g}$ of genus $g \geq 2$ is a hyperbolic group. Here, a random extension is one corresponding to a subgroup of either $\operatorname{Out}\left(F_{N}\right)$ or $\operatorname{Mod}\left(S_{g}\right)$ generated by $k$ independent random walks. Our main theorem is that a $k$-generated random subgroup of $\operatorname{Mod}\left(S_{g}\right)$ or $\operatorname{Out}\left(F_{N}\right)$ is free of rank $k$ and convex cocompact. More generally, we show that a $k$-generated random subgroup of a weakly hyperbolic group is free and undistorted.

\section{Introduction}

Let $F=F_{N}$ denote the free group of rank $N \geq 3$ and $S=S_{g}$ the closed, orientable surface of genus $g \geq 2$. Group extensions of both $F$ and $\pi_{1}(S)$ can be understood by investigating subgroups of their respective outer automorphism groups. For this, denote the outer automorphism group of $F$ by $\operatorname{Out}(F)$ and the mapping class group of $S$ by $\operatorname{Mod}(S)$.

For the surface $S$, there is the well-known Birman exact sequence [3]

$$
1 \rightarrow \pi_{1}(S) \rightarrow \operatorname{Mod}(S ; p) \stackrel{f}{\rightarrow} \operatorname{Mod}(S) \rightarrow 1
$$


where $\operatorname{Mod}(S ; p)$ denotes the group of mapping classes that fix the marked point $p \in S$ and $f: \operatorname{Mod}(S ; p) \rightarrow \operatorname{Mod}(S)$ is the surjective homomorphism that forgets this requirement. Given any finitely generated subgroup $\Gamma \leq \operatorname{Mod}(S)$, its preimage $E_{\Gamma}=f^{-1}(\Gamma)$ in $\operatorname{Mod}(S ; p)$ is a finitely generated group fitting into the sequence

$$
1 \rightarrow \pi_{1}(S) \rightarrow E_{\Gamma} \rightarrow \Gamma \rightarrow 1
$$

We say that $E_{\Gamma}$ is the surface group extension corresponding to $\Gamma \leq \operatorname{Mod}(S)$. Much work has gone into understanding what conditions on $\Gamma \leq \operatorname{Mod}(S)$ imply that the corresponding extension $E_{\Gamma}$ is hyperbolic. Such subgroups were introduced by Farb and Mosher as convex cocompact subgroups of the mapping class group [11] and have since become an active area of study. See for example [7, 16, 21, 22, 27] and Section 2.3 for details.

The situation for extensions of the free group $F$ is similar; by definition there is the short exact sequence

$$
1 \rightarrow F \rightarrow \operatorname{Aut}(F) \stackrel{f}{\rightarrow} \operatorname{Out}(F) \rightarrow 1
$$

where $f: \operatorname{Aut}(F) \rightarrow \operatorname{Out}(F)$ is now the induced quotient homomorphism. As before, a finitely generated subgroup $\Gamma \leq \operatorname{Out}(F)$ pulls back via $f$ to the corresponding free group extension $E_{\Gamma}=f^{-1}(\Gamma)$. Conditions on $\Gamma \leq \operatorname{Out}(F)$ which imply that the extension group $E_{\Gamma}$ is hyperbolic were recently given by Dowdall and the first author in [8]. See Section 2.4 for details.

In this note, we consider the following question:

Question 1.1. Given a random subgroup $\Gamma$ of either $\operatorname{Mod}(S)$ or $\operatorname{Out}(F)$, how likely is it that the corresponding extension group $E_{\Gamma}$ is hyperbolic?

By employing techniques developed by Maher and the second author in [26], we answer Question 1.1 by considering subgroups generated by random walks on either $\operatorname{Mod}(S)$ or $\operatorname{Out}(F)$. The point is that the references above characterize hyperbolicity of the extension group $E_{\Gamma}$ (for both $F$ and $\pi_{1}(S)$ ) solely in terms of the action of $\Gamma$ on a certain hyperbolic graph. This is precisely the situation considered in [26]. For this reason, we can treat both the cases of free group extensions and surface group extensions at once.

\subsection{Results}

To state our main theorem, we briefly introduce our model of random subgroups. Additional background on random walks is given in Section 2.1. 
First, let $X$ be a separable hyperbolic metric space and $G$ a countable group acting on $X$ by isometries. Two elements $g, h \in G$ which are loxodromic for the action on $X$ are independent if their quasi-axes determine four distinct endpoints on $\partial X$. A probability measure $\mu$ on $G$ is nonelementary if the semigroup generated by its support contains two independent loxodromic elements.

Now, let $\mu$ be a nonelementary probability measure on $G$ with respect to the action $G \curvearrowright X$ and consider $k$ independent random walks $\left(w_{n}^{(1)}\right)_{n \in \mathbb{N}}, \ldots,\left(w_{n}^{(k)}\right)_{n \in \mathbb{N}}$ whose increments are distributed according to $\mu$. For each $n \in \mathbb{N}$, we can consider the subgroup generated by the $n$th steps of our random walks,

$$
\Gamma(n)=\left\langle w_{n}^{(1)}, \ldots, w_{n}^{(k)}\right\rangle \leq G
$$

which we endow with the word metric coming from a given generating set.

Theorem 1.2. Let $G$ be a countable group with a nonelementary action by isometries on a separable hyperbolic space $X$. Let $\mu$ be a nonelementary probability measure on $G$ and fix $x_{0} \in X$. Then, the probability that the orbit map

$$
\begin{aligned}
\Gamma(n)=\left\langle w_{n}^{(1)}, \ldots, w_{n}^{(k)}\right\rangle & \rightarrow X, \\
g & \mapsto g \cdot x_{0}
\end{aligned}
$$

is a quasi-isometric embedding goes to 1 as $n \rightarrow \infty$. Moreover, the probability that the subgroup $\Gamma(n)$ is free of rank $k$ goes to 1 as $n \rightarrow \infty$.

Combining Theorem 1.2 with work of Farb-Mosher [11], Kent-Leininger [22], and Hamenstädt [16] (see Section 2.3) answers Question 1.1 for surface group extensions. Recall that $S$ is a closed surface of genus $\geq 2$.

Theorem 1.3 (Random surface group extensions). Let $\mu$ be a nonelementary probability measure on $\operatorname{Mod}(S)$ and let $\Gamma(n)=\left\langle w_{n}^{(1)}, \ldots, w_{n}^{(k)}\right\rangle$ denote the subgroup generated by the $n$th steps of $k$ independent random walks. Then the probability that $\Gamma(n)$ is free of rank $k$ and that the surface group extension $E_{\Gamma(n)}$ is hyperbolic goes to 1 as $n \rightarrow \infty$.

Proof. As discussed in Section 2.3, the mapping class group has a nonelementary action by isometries on the curve graph $\mathcal{C}(S)$. Theorem 1.2 then implies that the probability that the orbit map $\Gamma(n) \rightarrow \mathcal{C}(S)$ is a quasi-isometric embedding goes to 1 as $n \rightarrow \infty$. Since the extension $E_{\Gamma(n)}$ is hyperbolic whenever $\Gamma(n) \rightarrow \mathcal{C}(S)$ is a quasi-isometric embedding (Theorem 2.3), the theorem follows. 
Note that Theorem 1.3 implies that a random $k$-generated subgroup of $\operatorname{Mod}(S)$ is convex cocompact.

For extensions of free groups, we answer Question 1.1 by combining Theorem 1.2 with work of Dowdall and the first author [8].

Theorem 1.4 (Random free group extensions). Let $\mu$ be a nonelementary probability measure on $\operatorname{Out}(F)$ and let $\Gamma(n)=\left\langle w_{n}^{(1)}, \ldots, w_{n}^{(k)}\right\rangle$. Then the probability that $\Gamma(n)$ is free of rank $k$ and that the free group extension $E_{\Gamma(n)}$ is hyperbolic goes to 1 as $n \rightarrow \infty$.

Proof. As in Section 2.4, $\operatorname{Out}(F)$ has a nonelementary action by isometries on the hyperbolic graph $Y$. By Theorem 1.2, the probability that the orbit map $\Gamma(n) \rightarrow Y$ is a quasi-isometric embedding goes to 1 as $n \rightarrow \infty$. By Theorem 2.4, the extension $E_{\Gamma(n)}$ is hyperbolic whenever $\Gamma(n) \rightarrow Y$ is a quasi-isometric embedding, so the theorem follows.

As in the case of $\operatorname{Mod}(S)$, Theorem 1.4 implies that a random $k$-generated subgroup of $\operatorname{Out}(F)$ is convex cocompact, in the sense defined by Hamenstädt and Hensel [17].

Remark 1.5. When $k=1$, Theorem 1.3 is equivalent to the statement that the probability that $w_{n}$ is pseudo-Anosov goes to 1 as $n \rightarrow \infty$. Versions of this result were proved by Rivin [34] and Maher [25]. Likewise, Theorem 1.4 was also previously known in the special case where $k=1$, though by different methods. Indeed, Ilya Kapovich and Igor Rivin showed that for a random walk $\left(w_{n}\right)$ on $\operatorname{Out}(F)$ (with additional restrictions on the measure $\mu$ ), the probability that $w_{n}$ is atoroidal and fully irreducible goes to 1 as $n \rightarrow \infty$ [35]. (See Section 2.4 for definitions.)

\subsection{Further applications}

Thanks to the generality of Theorem 1.2, which is made possible by the general framework of [26], we can provide several other applications of interest. Following [26], we say that $G$ is weakly hyperbolic if $G$ admits a nonelementary action on a separable hyperbolic space $X$. We say that a random $k$-generated subgroup of $G$ has property $P$ if

$$
\mathbb{P}[\Gamma(n) \text { has } P] \rightarrow 1
$$

as $n \rightarrow \infty$. Here, as above, $\Gamma(n)=\left\langle w_{n}^{(1)}, \ldots, w_{n}^{(k)}\right\rangle$ denotes the subgroup of $G$ generated by the $n$th steps of $k$ independent random walks whose increments are distributed 
according to $\mu$. For any nonelementary measure $\mu$ on a finitely generated group $G$, the proof of Theorem 1.2 additionally yields the following corollary. First, recall that a finitely generated subgroup $\Gamma \leq G$ is undistorted if the inclusion $\Gamma \rightarrow G$ is a quasiisometric embedding with respect to some (any) word metrics on $\Gamma$ and $G$.

Corollary 1.6. Suppose that $G$ is finitely generated, weakly hyperbolic, and that $\mu$ is a nonelementary measure on $G$. Then for any $k \geq 1$ a random $k$-generated subgroup $G$ is free of rank $k$ and is undistorted in $G$.

We note that when $G$ itself is a hyperbolic group, Gilman, Miasnikov, and Osin have shown that a random $k$-generated subgroup of $G$ is free and undistorted [13]. The novelty of Corollary 1.6 is that it applies to a much larger class of groups; for example, mapping class groups, outer automorphism groups of free groups, right-angled Artin groups, acylindrically hyperbolic groups, and others. Our model of random subgroups appears in several other places in the literature: starting from Guivarc'h's random walk proof of the Tits alternative [15], it is explicitly formulated by Rivin [35], and Aoun [1], who proves that a random subgroup of a nonvirtually solvable linear group is free and undistorted. Moreover, Myasnikov and Ushakov [33] prove that random subgroups of pure braid groups are free, and provide applications of such results to cryptography.

As in the situation of the actions $\operatorname{Mod}(S) \curvearrowright \mathcal{C}(S)$ and $\operatorname{Out}(F) \curvearrowright Y$ (discussed in Sections 2.3 and 2.4), one is often particularly interested in those elements that act as loxodromic isometries (i.e., have positive translation length). It is immediate from Theorem 1.2 that, under the hypotheses of the theorem, every element of a random subgroup of $G$ is loxodromic.

In the special case of a right-angled Artin group $A(\Gamma)$, Kim and Koberda have introduced the extension graph $\Gamma^{e}$, a hyperbolic graph which admits a nonelementary action $A(\Gamma) \curvearrowright \Gamma^{e}$ [23]. The loxodromic isometries of $A(\Gamma)$ with respect to this action are precisely those $g \in A(\Gamma)$ with cyclic centralizers. In [24], the authors study the properties of purely loxodromic subgroups of $A(\Gamma)$, that is, the subgroups for which all nontrivial elements are loxodromic for the action $A(\Gamma) \curvearrowright \Gamma^{e}$, and show their resemblance to convex cocompact subgroups of mapping class groups. From Theorem 1.2, we have the following corollary.

Corollary 1.7. Let $\Gamma$ be a finite simplicial graph that does not decompose as a nontrivial join. Then for any $k \geq 1$ a $k$-generated random subgroup of $A(\Gamma)$ is free of rank $k$ and purely loxodromic. 


\section{Background}

\subsection{Random walks}

Let $\mu$ be a probability measure on a countable group $G$. In order to define a random walk on $G$, let us consider a probability space $(\Omega, \mathbb{P})$, and for each $n \in \mathbb{N}$, let $g_{n}: \Omega \rightarrow$ $G$ be a $G$-valued random variable such that the $g_{n}$ 's are independent and identically distributed with distribution $\mu$. We define the $n$th step of the random walk to be the variable $w_{n}: \Omega \rightarrow G$

$$
w_{n}:=g_{1} g_{2} \cdots g_{n}
$$

The sequence $\left(w_{n}\right)_{n \in \mathbb{N}}$ is called a sample path of the random walk. We denote as $\mu_{n}$ the distribution of $w_{n}$, which equals the $n$-fold convolution of $\mu$ with itself. That is,

$$
\mu_{n}(x)=\mathbb{P}\left[w_{n}=x\right]=\sum_{x=g_{1} \ldots g_{n}} \mu\left(g_{1}\right) \cdots \mu\left(g_{n}\right),
$$

which describes the probability that the $n$th step of the random walk lands at $x \in G$.

Moreover, the reflected measure $\check{\mu}$ is defined as $\check{\mu}(g):=\mu\left(g^{-1}\right)$. We note that for the random walk $\left(w_{n}\right)$, the distribution of $w_{n}^{-1}$, the inverse of the $n$th step of the random walk, is given by $(\breve{\mu})_{n}=\left(\check{\mu}_{n}\right)$.

Just as a random walk allows one to speak of a "random" element of $G$, we can use $k$ independent random walks to model a ( $k$-generator) random subgroup of $G$. In details, fix a probability measure $\mu$ on $G$ and let $\left(w_{n}^{(1)}\right)_{n \in \mathbb{N}}, \ldots,\left(w_{n}^{(k)}\right)_{n \in \mathbb{N}}$ be $k$ independent random walks each of whose increments are distributed according to $\mu$. For each $n \in \mathbb{N}$, we can consider the subgroup generated by the nth steps of our sample paths,

$$
\Gamma(n)=\left\langle w_{n}^{(1)}, \ldots, w_{n}^{(k)}\right\rangle \leq G
$$

For example, if $G$ is finitely generated and $\mu$ is the uniform measure supported on a finite, symmetric generating set $S$ for $G$, then $\Gamma(n)$ is the subgroup generated by selecting $k$ (unreduced) words of length $n$ in the basis $S$ uniformly at random.

We remark that this model for a random subgroup of $G$ appears several places in the literature; see, for example, [1, 13, 15, 33, 35].

\subsection{Actions on hyperbolic spaces}

Now suppose that $X$ is a separable hyperbolic space and that $G$ acts on $X$ by isometries. The action $G \curvearrowright X$ is nonelementary if there are $g, h \in G$ which act loxodromically 
on $X$ and whose fixed point sets on the Gromov boundary of $X$ are disjoint. Recall that an isometry $g$ of $X$ is loxodromic if it has positive translation length on $X$, that is, $\liminf _{n \rightarrow \infty} d\left(x_{0}, g x_{0}\right) / n>0$ for some $x_{0} \in X$. A probability measure $\mu$ on $G$ is said to be nonelementary with respect to the action $G \curvearrowright X$ if the semigroup generated by the support of $\mu$ contains two independent loxodromic elements. In this note, we are interested in the behavior of the image of a random walk $\left(w_{n}\right)$ under an orbit map $G \rightarrow X$. Hence, we fix once and for all a basepoint $x_{0} \in X$ and consider the orbit map $G \rightarrow X$ given by $g \mapsto g x_{0}$.

Central to Maher and Tiozzo's study of random walks on $G$ is the notion of shadows, which we now summarize. First, recall that for $x, y, z \in X$, the Gromov product, denoted $(y \cdot z)_{X}$, is defined as

$$
(y \cdot z)_{x}=\frac{1}{2}(d(x, y)+d(x, z)-d(y, z)) .
$$

When $X$ is hyperbolic, the Gromov product $(y \cdot z)_{z}$ coarsely agrees with the distance from $x$ to any geodesic between $y$ and $x$. See [5] for details.

Given $x_{0}, X \in X$ and $R \geq 0$, the shadow $S_{X_{0}}(X, R) \subset X$ is by definition the set

$$
S_{X_{0}}(x, R)=\left\{y \in X:(x \cdot y)_{X_{0}} \geq d_{X}\left(x_{0}, x\right)-R\right\} .
$$

When $R<0$, we declare that $S_{X_{0}}(x, R)=\emptyset$. The distance parameter of the shadow $S_{X_{0}}(x, R)$ is the quantity $d\left(x_{0}, x\right)-R$, which is coarsely equal to the distance from $x_{0}$ to the shadow. It follows easily from hyperbolicity of $X$ that there is a constant $C$, depending only on the hyperbolicity constant of $X$, such that

$$
\left(X \backslash S_{X_{0}}(x, R)\right) \subset S_{X}\left(x_{0}, d\left(x_{0}, x\right)-R+C\right) .
$$

See [26] for details.

We will need two additional results from [26]. The first roughly states that the measure of a shadow decays to zero as the distance parameter goes to infinity. For the precise statement, set

$$
\operatorname{Sh}\left(x_{0}, r\right)=\left\{S_{x_{0}}\left(g x_{0}, R\right): g \in G \text { and } d\left(x_{0}, g x_{0}\right)-R \geq r\right\}
$$

This is the set of shadows based at $x_{0}$ and centered at points in the orbit of $x_{0}$ with distance parameter at least $r$. The following is [26, Corollary 5.3]. 
Lemma 2.1 (Maher-Tiozzo). Let $G$ be a countable group which acts by isometries on a separable hyperbolic space $X$, and let $\mu$ be a nonelementary probability distribution on $G$. Then there is a function $f(r)$, with $f(r) \rightarrow 0$ as $r \rightarrow \infty$ such that for all $n$

$$
\sup _{S \in S h\left(x_{0}, r\right)}\left\{\begin{array}{l}
\mu_{n}(S) \\
\check{\mu}_{n}(S)
\end{array}\right\} \leq f(r) .
$$

Finally, we will need the fact that a random walk on $G$ whose increments are distributed according to a nonelementary measure has positive drift in $X$. This is [26, Theorem 1.2].

Theorem 2.2 (Maher-Tiozzo). Let $G$ be a countable group which acts by isometries on a separable hyperbolic space $X$, and let $\mu$ be a nonelementary probability distribution on $G$. Fix $x_{0} \in X$. Then, there is a constant $L>0$ such that for almost every sample path

$$
\liminf _{n \rightarrow \infty} \frac{d\left(x_{0}, w_{n} x_{0}\right)}{n}=L>0
$$

The constant $L>0$ in Theorem 2.2 is called the drift of the random walk $\left(w_{n}\right)$.

We remark that the study of random walks on Gromov hyperbolic groups goes back to Kaimanovich [19], who among other things proved that the Poisson boundary of such random walks coincides with the Gromov boundary. Note that in this classical case the space on which $G$ acts is proper, hence the positive drift statement follows simply from nonamenability of the group. More recently, authors have started considering random walks for groups acting on nonproper spaces; in particular, Rivin [34] and Maher [25] for the curve graph, and in greater generality Calegari and Maher [6] and Sisto [36].

\subsection{Hyperbolic extensions of surface groups}

Here, we briefly recall some background on convex cocompact subgroups of mapping class groups. See [11,22] for details.

Fix $S=S_{g}$, a closed, orientable surface of genus $g \geq 2$. Associated to $S$ are its mapping class group $\operatorname{Mod}(S)$, its Teichmüller space $\mathcal{T}(S)$ (considered with the Teichmüller metric), and its curve graph $\mathcal{C}(S)$. We refer the reader to [10] for definitions and background on these standard objects in surface topology. We recall that there are natural actions $\operatorname{Mod}(S) \curvearrowright \mathcal{T}(S)$ and $\operatorname{Mod}(S) \curvearrowright \mathcal{C}(S)$; the former given by remarking and the latter 
given by the action of mapping classes on isotopy classes of simple closed curves (see remarks following Theorem 2.3 for details). While the action $\operatorname{Mod}(S) \curvearrowright \mathcal{T}(S)$ is properly discontinuous, $\mathcal{T}(S)$ is not negatively curved [30, 32]. On the other hand, $\mathcal{C}(S)$ is a locally infinite graph and the action $\operatorname{Mod}(S) \curvearrowright \mathcal{C}(S)$ has large vertex stabilizers, but $\mathcal{C}(S)$ is hyperbolic by the foundational work of Masur and Minsky [31]. Much can be learned about the coarse geometry of $\operatorname{Mod}(S)$ by studying its action on both $\mathcal{T}(S)$ and $\mathcal{C}(S)$ in conjunction with the equivalent coarsely Lipschitz map $\mathcal{T}(S) \rightarrow \mathcal{C}(S)$, which associates to each marked hyperbolic surface its collection of shortest curves.

In [11], Farb and Mosher introduced convex cocompact subgroups of $\operatorname{Mod}(S)$ as those finitely generated subgroups $\Gamma \leq \operatorname{Mod}(S)$ for which the orbit $\Gamma \cdot X \subset \mathcal{T}(S)$ of some $X \in \mathcal{T}(S)$ is quasiconvex with respect to the Teichmüller metric. Our interest in convex cocompact subgroups of $\operatorname{Mod}(S)$ comes from their connection to hyperbolicity of surface group extensions. This connection is summarized in the following theorem. For additional characterizations of convex cocompactness, see [9, 22].

Theorem 2.3 (Farb-Mosher, Kent-Leininger, Hamenstädt). Let $\Gamma$ be a finitely generated subgroup of $\operatorname{Mod}(S)$. Then the following are equivalent:

(1) $\Gamma$ is convex cocompact,

(2) the orbit map $\Gamma \rightarrow \mathcal{C}(S)$ is a quasi-isometric embedding, and

(3) the extension $E_{\Gamma}$ is hyperbolic.

In Theorem 2.3, the implication (3) $\Longrightarrow(1)$ is due to Farb-Mosher, as is the converse (1) $\Longrightarrow(3)$ when $\Gamma$ is free [11]. The general case of (1) $\Longrightarrow(3)$ is due to Hamenstädt [16]. Finally, the equivalence (1) $\Longleftrightarrow(2)$ is due to Kent-Leininger [22] and, independently, Hamenstädt [16]. To show that random subgroups of $\operatorname{Mod}(S)$ induce hyperbolic extensions of $\pi_{1}(S)$ (Theorem 1.3), we only need the implication (2) $\Longrightarrow$ (3) in Theorem 2.3.

Recall that the curve graph $\mathcal{C}(S)$ is the graph whose vertices are isotopy classes of essential simple closed curve and whose edges join vertices that have disjoint representatives on $S$. As stated above, Masur-Minsky showed that $\mathcal{C}(S)$ is hyperbolic and that the loxodromic elements of the action $\operatorname{Mod}(S) \curvearrowright \mathcal{C}(S)$, that is, those elements with positive translation length, are precisely the pseudo-Anosov mapping classes [31]. From this, it easily follows that the action $\operatorname{Mod}(S) \curvearrowright \mathcal{C}(S)$ is nonelementary. In fact, it is known that the action satisfies the much stronger property of being acylindrical [4], however, we will not need this fact here. 


\subsection{Hyperbolic extensions of free groups}

Here, we recall some background on hyperbolic extensions of free groups. See [8] for additional detail.

Fix $F=F_{N}$, the free group of $\operatorname{rank} N \geq 3$ and denote by $\operatorname{Out}(F)$ its outer automorphism group. Although we will only require the statement of Theorem 2.4, we recall that $f \in \operatorname{Out}(F)$ is fully irreducible if no positive power of $f$ fixes any conjugacy class of any free factor of $F$. Also, $f \in \operatorname{Out}(F)$ is atoroidal if no positive power fixes any conjugacy class of elements of $F$. If the extension $E_{\Gamma}$ of $\Gamma \leq \operatorname{Out}(F)$ is hyperbolic, then it is necessarily the case that each infinite-order element of $\Gamma$ is atoroidal.

In [8], Dowdall and the first author study conditions on $\Gamma \leq \operatorname{Out}(F)$ which imply that the extension $E_{\Gamma}$ is hyperbolic. These conditions are given in terms of the action of $\operatorname{Out}(F)$ on the free factor complex $\mathcal{F}$, an $\operatorname{Out}(F)$-complex introduced by Hatcher and Vogtmann [18] and proved to be hyperbolic by Bestvina and Feighn [2]. For our application, it is most convenient to work with a version of the intersection graph, an Out $(F)$ graph introduced by Kapovich and Lustig [20] and further studied by Mann [28] and Mann and Reynolds [29].

In particular, Mann and Reynolds show that there is a graph $Y$ with a simplicial action $\operatorname{Out}(F) \curvearrowright Y$ satisfying the following properties: (1) $Y$ is hyperbolic, (2) there is an $\operatorname{Out}(F)$-equivariant map $\mathcal{F} \rightarrow Y$ which is coarsely Lipschitz, and (3) $f \in \operatorname{Out}(F)$ is loxodromic for the action $\operatorname{Out}(F) \curvearrowright Y$ if and only if $f$ is atoroidal and fully irreducible. See $[28,29]$ for details. The following theorem is essentially Theorem 9.2 of [8].

Theorem 2.4 (Dowdall-Taylor). Let $\Gamma$ be a finitely generated subgroup of Out $(F)$. Suppose that the orbit map $\Gamma \rightarrow Y$ is a quasi-isometric embedding for some $x_{0} \in Y$. Then the corresponding extension $E_{\Gamma}$ is hyperbolic.

Proof. Since there is a coarsely Lipschitz, equivariant map $\mathcal{F} \rightarrow Y$ (by property (2) of $Y$ ), there is a $z_{0} \in \mathcal{F}$ such that the orbit map $\Gamma \rightarrow \mathcal{F}$ based at $z_{0}$ is a quasi-isometric embedding. Moreover, since infinite-order elements of $\Gamma$ are loxodromic with respect to the action $\operatorname{Out}(F) \curvearrowright Y$, these elements must be atoroidal (by property (3) of $Y$ ). We now apply [8, Theorem 1.1] which states that $E_{\Gamma}$ is hyperbolic if every infinite-order element of $\Gamma$ is atoroidal and the orbit map $\Gamma \rightarrow \mathcal{F}$ into the free factor complex is a quasi-isometric embedding.

Just as in the situation of the mapping class group acting on the curve graph, it follows that the action $\operatorname{Out}(F) \curvearrowright Y$ is nonelementary, that is, there exists a pair of 
loxodromic elements with no common fixed points on $\partial Y$. In fact, according to MannReynolds [28, 29], the action $\operatorname{Out}(F) \curvearrowright Y$ satisfies the stronger property of being weakly properly discontinuous.

\section{Proof of Theorem 1.2}

We begin by providing conditions for when the orbit map from a $k$-generated group into a hyperbolic space is a quasi-isometric embedding. We require the following well-known lemma; see, for example, [12-14].

Lemma 3.1. Let $X$ be a $\delta$-hyperbolic metric space with points $p_{0}, \ldots, p_{n}$ satisfying

$$
\min \left\{d\left(p_{i-1}, p_{i}\right), d\left(p_{i}, p_{i+1}\right)\right\} \geq 2\left(p_{i-1} \cdot p_{i+1}\right) p_{i}+18 \delta+1
$$

Then, $d\left(p_{0}, p_{n}\right) \geq n$.

Lemma 3.1 easily implies the following:

Lemma 3.2. Let $X$ be a $\delta$-hyperbolic space and, for $1 \leq i \leq k$, let $g_{i} \in \operatorname{Isom}(X)$ be isometries of $X$ such that for some $x_{0} \in X$ we have

$$
d\left(x_{0}, g_{i} x_{0}\right) \geq 2\left(g_{j}^{ \pm 1} x_{0} \cdot g_{l}^{ \pm 1} x_{0}\right)_{x_{0}}+18 \delta+1
$$

for all $1 \leq i, j, l \leq k$ except when $j=l$ and the exponent on the $g_{j}$ and $g_{l}$ are the same. Set $\Gamma=\left\langle g_{1} \ldots, g_{k}\right\rangle$. Then the orbit map $\Gamma \rightarrow X$ given by $g \mapsto g x_{0}$ is a quasi-isometric embedding, and $\Gamma$ is free of rank $k$.

Proof. As the orbit map $\Gamma \rightarrow X$ is always coarsely Lipschitz, it suffices to prove that for any $g \in \Gamma$,

$$
|g|_{\Gamma} \leq d\left(x_{0}, g x_{0}\right)
$$

To see this, write $g=s_{0} \cdots s_{n}$ as a reduced word where $s_{i} \in\left\{g_{0}^{ \pm 1}, \ldots, g_{k}^{ \pm 1}\right\}$ and $n=|g|_{\Gamma}$. Letting $p_{i}=\left(s_{0} s_{1} \cdots s_{i}\right) x_{0}$, we note that by Lemma 3.1 it suffices to show that Inequality (2) holds for these points. Observe that since the action of $\Gamma$ is by isometries on $X$,

$$
\begin{aligned}
\min \left\{d\left(p_{i-1}, p_{i}\right), d\left(p_{i}, p_{i+1}\right)\right\} & =\min \left\{d\left(x_{0}, s_{i} x_{0}\right), d\left(x_{0}, s_{i+1} x_{0}\right)\right\} \\
& \geq 2\left(s_{i}^{-1} x_{0} \cdot s_{i+1} x_{0}\right)_{x_{0}}+18 \delta+1 \\
& =2\left(p_{i-1} \cdot p_{i+1}\right)_{p_{i}}+18 \delta+1,
\end{aligned}
$$


where the first inequality holds by (3) and the fact that $s_{i} \neq s_{i+1}^{-1}$. This completes the proof.

Lemma 3.3. Let $G$ be a countable group acting by isometries on a hyperbolic space $X$. Let $\mu$ be a nonelementary probability measure on $G$, fix $x_{0} \in X$, and let $l: \mathbb{N} \rightarrow \mathbb{N}$ be any function with $l(n) \rightarrow \infty$ as $n \rightarrow \infty$. Suppose that $\left(w_{n}\right)$ and $\left(u_{n}\right)$ are independent random walks on $G$ whose increments are distributed according to $\mu$. Then

$$
\mathbb{P}\left[\left(w_{n}^{ \pm 1} x_{0} \cdot u_{n}^{ \pm 1} x_{0}\right)_{x_{0}} \leq l(n)\right] \rightarrow 1,
$$

as $n \rightarrow \infty$.

Proof. First note that since $\left(w_{n}\right)$ and $\left(u_{n}\right)$ are independent random walks with increments distributed according to $\mu$, both $w_{n}$ and $u_{n}$ have distribution $\mu_{n}$. Moreover, $w_{n}^{-1}$ and $u_{n}^{-1}$, the inverses of the $n$th steps, have distribution $\check{\mu}_{n}$ as noted at the beginning of Section 2.1. Since the proofs of (4) in each of the four possible cases are identical, we show

$$
\mathbb{P}\left[\left(w_{n} X_{0} \cdot u_{n}^{-1} x_{0}\right)_{x_{0}} \leq l(n)\right] \rightarrow 1,
$$

as $n \rightarrow \infty$.

By setting $R_{n}=d\left(x_{0}, u_{n} x_{0}\right)-l(n)$, we have

$$
\mathbb{P}\left[\left(w_{n} x_{0} \cdot u_{n}^{-1} x_{0}\right)_{x_{0}} \leq l(n)\right]=1-\mathbb{P}\left[w_{n} x_{0} \in S_{X_{0}}\left(u_{n}^{-1} x_{0}, R_{n}\right)\right],
$$

where the shadow $S_{X_{0}}\left(u_{n} X_{0}, R_{n}\right)$ has distance parameter $l(n)$. As $w_{n}$ and $u_{n}^{-1}$ are independent with distributions $\mu_{n}$ and $\check{\mu}_{n}$, respectively, we have that

$$
\begin{aligned}
\mathbb{P}\left[w_{n} X_{0} \in S_{X_{0}}\left(u_{n}^{-1} X_{0}, R_{n}\right)\right] & =\sum_{g \in G} \mathbb{P}\left[w_{n} X_{0} \in S_{X_{0}}\left(u_{n}^{-1} x_{0}, R_{n}\right) \mid u_{n}^{-1}=g\right] \cdot \check{\mu}_{n}(g) \\
& =\sum_{g \in G} \mu_{n}\left(S_{X_{0}}\left(g x_{0}, R_{n}\right)\right) \check{\mu}_{n}(g) \\
& \leq f(l(n))
\end{aligned}
$$

where the last inequality uses the decay of shadows (Lemma 2.1). Since $f(l(n)) \rightarrow 0$ as $n \rightarrow \infty$, the lemma follows.

Lemma 3.4. Let $G$ be a countable group acting by isometries on a hyperbolic space $X$. Let $\mu$ be a nonelementary probability measure on $G$ and fix $x_{0} \in X$. Further, let $l: \mathbb{N} \rightarrow \mathbb{N}$ be any function with $l(n) \rightarrow \infty$ as $n \rightarrow \infty$ and $\lim \sup \frac{l(n)}{n}<\frac{L}{2}$, where $L$ is the drift of $\left(w_{n}\right)$. 
Suppose that $\left(w_{n}\right)$ is a random walk on $G$ whose increments are distributed according to $\mu$. Then

$$
\mathbb{P}\left[\left(w_{n} x_{0} \cdot w_{n}^{-1} x_{0}\right)_{x_{0}} \leq l(n)\right] \rightarrow 1,
$$

as $n \rightarrow \infty$.

Proof. We follow the argument in [26]. For each $n$, let $m:=\left\lfloor\frac{n}{2}\right\rfloor$, and we can write $w_{n}=w_{m} u_{m}$, with $u_{m}:=w_{m}^{-1} w_{n}=g_{m+1} \cdots g_{n}$. Note that the random walks $w_{m}$ and $u_{m}$ are independent, and $u_{m}$ has distribution $\mu_{n-m}$. We first claim that

$$
\mathbb{P}\left[\left(w_{n} x_{0} \cdot w_{m} x_{0}\right)_{x_{0}} \geq l(n)\right] \rightarrow 1 \quad \text { as } n \rightarrow \infty .
$$

Proof of claim. Indeed,

$$
\begin{aligned}
\mathbb{P}\left[\left(w_{n} x_{0} \cdot w_{m} x_{0}\right)_{x_{0}} \geq l(n)\right] & =\mathbb{P}\left[\left(u_{m} x_{0} \cdot x_{0}\right)_{w_{m}^{-1} x_{0}} \geq l(n)\right] \\
& =\mathbb{P}\left[u_{m} x_{0} \in S_{w_{m}^{-1} x_{0}}\left(x_{0}, R\right)\right],
\end{aligned}
$$

where $R:=d\left(x_{0}, w_{m}^{-1} x_{0}\right)-l(n)$. Using Observation (1), we note that

$$
\left(X \backslash S_{w_{m}^{-1} X_{0}}\left(X_{0}, R\right)\right) \subset S_{X_{0}}\left(w_{m}^{-1} X_{0}, R_{n}\right),
$$

where $R_{n}=l(n)+C$, and $C$ depends only on the hyperbolicity constant of $X$. This implies that

$$
\mathbb{P}\left[\left(w_{n} x_{0} \cdot w_{m} x_{0}\right)_{x_{0}} \geq l(n)\right] \geq 1-\mathbb{P}\left[u_{m} x_{0} \in S_{X_{0}}\left(w_{m}^{-1} x_{0}, R_{n}\right)\right]
$$

Further, using independence of $w_{m}$ and $u_{m}$, we see

$$
\begin{aligned}
\mathbb{P}\left[u_{m} x_{0} \in S_{X_{0}}\left(w_{m}^{-1} x_{0}, R_{n}\right)\right] & =\sum_{g \in G} \mathbb{P}\left[u_{m} X_{0} \in S_{X_{0}}\left(w_{m}^{-1} x_{0}, R_{n}\right) \mid w_{m}=g\right] \cdot \mu_{m}(g) \\
& =\sum_{g \in G} \mu_{n-m}\left(S_{X_{0}}\left(g^{-1} X_{0}, R_{n}\right)\right) \mu_{m}(g)
\end{aligned}
$$

Let us now pick $\epsilon>0$ such that $\lim \sup \frac{l(n)+\epsilon n}{n}<\frac{L}{2}$, where $L$ is the drift of $\left(w_{n}\right)$; then by considering in Equation (5) only the $g$ such that $d\left(x_{0}, g^{-1} x_{0}\right) \geq l(n)+\epsilon n$, and using the estimate for the distance parameter of $S_{X_{0}}\left(g^{-1} x_{0}, R_{n}\right)$ we obtain

$$
\mathbb{P}\left[u_{m} x_{0} \in S_{x_{0}}\left(w_{m}^{-1} x_{0}, R_{n}\right)\right] \leq f(\epsilon n-C)+\mathbb{P}\left[d\left(x_{0}, w_{m} x_{0}\right) \leq l(n)+\epsilon n\right]
$$

The first term tends to 0 by the decay of shadows (Lemma 2.1) and the second because of linear progress (Theorem 2.2). This proves the claim. 
We return to the proof of Lemma 3.4. As in the proof of the claim, replacing $w_{n}$ with $w_{n}^{-1}$ and $w_{m}$ with $w_{n}^{-1} w_{m}=u_{m}^{-1}$ it follows that

$$
\mathbb{P}\left[\left(w_{n}^{-1} x_{0} \cdot w_{n}^{-1} w_{m} x_{0}\right)_{X_{0}} \geq l(n)\right] \rightarrow 1 \quad \text { as } n \rightarrow \infty .
$$

Then, by Lemma 3.4 (using that $w_{m}$ and $u_{m}$ are independent)

$$
\mathbb{P}\left[\left(w_{m} x_{0} \cdot w_{n}^{-1} w_{m} x_{0}\right)_{x_{0}} \leq l(n)-3 \delta\right] \rightarrow 1 \quad \text { as } n \rightarrow \infty .
$$

Finally, by $\delta$-hyperbolicity (Lemma 3.5 below),

$$
\mathbb{P}\left[\left(w_{n} x_{0} \cdot w_{n}^{-1} x_{0}\right)_{x_{0}} \leq l(n)+2 \delta\right] \rightarrow 1 \quad \text { as } n \rightarrow \infty .
$$

This completes the proof.

The following lemma was used in the proof of Lemma 3.4. It appears as Lemma 5.9 in [26], but is proved here for convenience to the reader.

Lemma 3.5 (Fellow traveling is contagious). Suppose that $X$ is a $\delta$-hyperbolic space with basepoint $x_{0}$ and suppose that $A \geq 0$. If $a, b, c, d \in X$ are points of $X$ with $(a \cdot b)_{x_{0}} \geq A$, $(c \cdot d)_{X_{0}} \geq A$, and $(a \cdot c)_{X_{0}} \leq A-3 \delta$. Then $(b \cdot d)_{X_{0}}-2 \delta \leq(a \cdot c)_{X_{0}} \leq(b \cdot d)_{X_{0}}+2 \delta$.

Proof. By hyperbolicity, $(a \cdot c)_{X_{0}} \geq \min \left\{(a \cdot b)_{X_{0}},(b \cdot c)_{X_{0}}\right\}-\delta$. Since $(a \cdot c)_{X_{0}} \leq(a \cdot b)_{X_{0}}-3 \delta$, it must be that $(a \cdot c)_{x_{0}} \geq(b \cdot c)_{x_{0}}-\delta$. Exactly, the same reasoning using the inequality $(a \cdot c)_{X_{0}} \geq \min \left\{(a \cdot d)_{X_{0}},(d \cdot c)_{X_{0}}\right\}-\delta$ gives that $(a \cdot c)_{X_{0}} \geq(a \cdot d)_{X_{0}}-\delta$. Hence, $(a \cdot d)_{X_{0}} \leq$ $(a \cdot c)_{X_{0}}+\delta \leq(a \cdot b)_{x_{0}}-2 \delta$.

Another application of hyperbolicity yields $(a \cdot d)_{x_{0}} \geq \min \left\{(a \cdot c)_{x_{0}},(c \cdot d)_{x_{0}}\right\}$ $-\delta=(a \cdot c)_{X_{0}}-\delta$. Combining these facts we have

$$
\begin{aligned}
(b \cdot d)_{X_{0}} & \geq \min \left\{(a \cdot b)_{X_{0}},(a \cdot d)_{X_{0}}\right\}-\delta \\
& =(a \cdot d)_{X_{0}}-\delta \\
& \geq(a \cdot c)_{X_{0}}-2 \delta .
\end{aligned}
$$


To prove the reverse inequality, first note that the inequality $(a \cdot d)_{x_{0}} \leq$ $(a \cdot b)_{x_{0}}-2 \delta$ obtained above implies that $(a \cdot d)_{x_{0}} \geq \min \left\{(a \cdot b)_{X_{0}},(b \cdot d)_{x_{0}}\right\}-\delta=(b \cdot d)_{x_{0}}-\delta$. Then

$$
\begin{aligned}
(a \cdot c)_{X_{0}} & \geq \min \left\{(a \cdot d)_{X_{0}},(d \cdot c)_{X_{0}}\right\}-\delta \\
& =(a \cdot d)_{X_{0}}-\delta \\
& \geq(b \cdot d)_{X_{0}}-2 \delta,
\end{aligned}
$$

as required.

We can now complete the proof of Theorem 1.2.

Proof of Theorem 1.2. Fix $x_{0} \in X$ and set $\Gamma(n)=\left\langle w_{n}^{(1)}, \ldots w_{n}^{(k)}\right\rangle$. By Lemma 3.2, the probability that $\Gamma(n) \rightarrow X$ is a quasi-isometric embedding is bounded below by the probability that

$$
d\left(x_{0}, w_{n}^{(i)} x_{0}\right) \geq 2\left(\left(w_{n}^{(j)}\right)^{ \pm 1} x_{0} \cdot\left(w_{n}^{(l)}\right)^{ \pm 1} x_{0}\right)_{X_{0}}+18 \delta+1
$$

for all choices of indices $1 \leq i, j, l \leq k$, excluding the cases that produce terms involving the Gromov product of a point with itself.

It is easily verified that the probability of (6) goes to 1 as $n \rightarrow \infty$. Indeed, by Theorem 2.2,

$$
\mathbb{P}\left[d\left(x_{0}, w_{n}^{(i)} x_{0}\right) \geq L^{\prime} n\right] \rightarrow 1
$$

as $n \rightarrow \infty$, for each $1 \leq i \leq k$ and any $L^{\prime}<L$, where $L$ is the drift of the random walks. Moreover, combining Lemmas 3.3 and 3.4, we see that for $j \neq l$,

$$
\mathbb{P}\left[\left(\left(w_{n}^{(j)}\right)^{ \pm 1} x_{0} \cdot\left(w_{n}^{(l)}\right)^{ \pm 1} X_{0}\right)_{X_{0}} \leq l(n)\right] \rightarrow 1
$$

and for each $1 \leq j \leq k$,

$$
\mathbb{P}\left[\left(w_{n}^{(j)} X_{0} \cdot\left(w_{n}^{(j)}\right)^{-1} X_{0}\right)_{X_{0}} \leq l(n)\right] \rightarrow 1,
$$

for any function $l(n)$ with $l(n) \rightarrow \infty$ as $n \rightarrow \infty$ and $\lim \sup \frac{l(n)}{n}<\frac{1}{2} L$. Additionally choosing $l(n)$ so that $2 l(n)+18 \delta+1<L n$ completes the proof.

\section{Acknowledgements}

We would like to thank Joseph Maher for several useful conversations and the anonymous referee for helpful comments. 


\section{Funding}

The first-named author is partially supported by NSF DMS-1204592.

\section{References}

[1] Aoun, R. "Random subgroups of linear groups are free." Duke Mathematical Journal 160, no. 1 (2011): 117-73.

[2] Bestvina, M. and M. Feighn. "Hyperbolicity of the complex of free factors." Advances in Mathematics 256 (2014): 104-55.

[3] Birman, J. S. "Mapping class groups and their relationship to braid groups." Communications on Pure and Applied Mathematics 22, no. 2 (1969): 213-38.

[4] Bowditch, B. H. "Tight geodesics in the curve complex." Inventiones Mathematicae 171, no. 2 (2008): 281-300.

[5] Bridson, M. R. and A. Haefliger. Metric Spaces of Non-positive Curvature, vol. 319. Berlin: Springer, 2009.

[6] Calegari, D. and J. Maher. "Statistics and compression of scl." Ergodic Theory and Dynamical Systems 35, no. 1 (2015): 64-110.

[7] Dowdall, S., R. P. Kent, IV, and C. J. Leininger. "Pseudo-Anosov subgroups of fibered 3-manifold groups." Groups, Geometry, and Dynamics 8, no. 4 (2014): 1247-82.

[8] Dowdall, S. and S. J. Taylor. "Hyperbolic extensions of free groups." (2014): preprint arXiv:1406.2567.

[9] Durham, M. G. and S. J. Taylor. "Convex cocompactness and stability in mapping class groups." Algebraic and Geometric Topology (2014), to appear.

[10] Farb, B. and D. Margalit. A Primer on Mapping Class Groups. Princeton Mathematical Series 49. Princeton, NJ: Princeton University Press, 2012.

[11] Farb, B. and L. Mosher. "Convex cocompact subgroups of mapping class groups." Geometry and Topology 6, no. 1 (2002): 91-152 (electronic).

[12] Ghys, E. and P. de la Harpe. Sur les groupes hyperboliques d'apres Mikhael Gromov. Basel: Birkhauser, 1990.

[13] Gilman, R., A. Miasnikov, and D. Osin. "Exponentially generic subsets of groups." Illinois Journal of Mathematics 54, no. 1 (2010): 371-88.

[14] Gromov, M. Hyperbolic Groups. Berlin: Springer, 1987.

[15] Guivarc'h, Y. "Produits de matrices aleatoires et applications aux proprietes geometriques des sous-groupes du groupe lineaire." Ergodic Theory and Dynamical Systems 10, no. 03 (1990): 483-512.

[16] Hamenstädt, U. "Word hyperbolic extensions of surface groups." (2005): preprint arxiv:0807.4891v2.

[17] Hamenstädt, U. and S. Hensel. "Convex cocompact subgroups of $\operatorname{Out}\left(F_{n}\right)$. " (2014): preprint arXiv:1411.2281.

[18] Hatcher, A. and K. Vogtmann. "The complex of free factors of a free group." Quarterly Journal of Mathematics 49, no. 196 (1998): 459-68. 
[19] Kaimanovich, V. A. "The Poisson boundary of hyperbolic groups." Comptes Rendus de l'Académie des Sciences - Series I - Mathematics 318, no. 1 (1994): 59-64.

[20] Kapovich, I. and M. Lustig. "Geometric intersection number and analogues of the curve complex for free groups." Geometry and Topology 13, no. 3 (2009): 1805-33.

[21] Kent, R. P., IV and C. J. Leininger. "Subgroups of Mapping Class Groups from the Geometrical Viewpoint." In the Tradition of Ahlfors-Bers. IV, 119-41. Contemporary Mathematics 432. Providence, RI: American Mathematical Society, 2007.

[22] Kent, R. P., IV and C. J. Leininger. "Shadows of mapping class groups: capturing convex cocompactness." Geometric and Functional Analysis 18, no. 4 (2008): 1270-325.

[23] Kim, S-h and T. Koberda. "Embeddability between right-angled Artin groups." Geometry and Topology 17, no. 1 (2013): 493-530.

[24] Koberda, T., J. Mangahas, and S. J. Taylor. "The geometry of purely loxodromic subgroups of right-angled Artin groups." (2014): preprint arXiv:1412.3663.

[25] Maher, J. "Random walks on the mapping class group." Duke Mathematical Journal 156, no. 3 (2011): 429-68.

[26] Maher, J. and G. Tiozzo. "Random walks on weakly hyperbolic groups." (2014): preprint arXiv:1410.4173.

[27] Mangahas, J. and S. J. Taylor. "Convex cocompactness in mapping class groups via quasiconvexity in right-angled Artin groups." (2013): preprint arXiv:1306.5278.

[28] Mann, B. “Some hyperbolic Out $\left(F_{n}\right)$-graphs and nonunique ergodicity of very small $F_{n}$-trees." PhD thesis, The University of Utah, 2014.

[29] Mann, B. and P. Reynolds. In preparation.

[30] Masur, H. “On a class of geodesics in Teichmuller space." Annals of Mathematics 102, no. 2 (1975): 205-21.

[31] Masur, H. A. and Y. N. Minsky. "Geometry of the complex of curves. I. Hyperbolicity." Inventiones Mathematicae 138, no. 1 (1999): 103-49.

[32] Masur, H. A. and M. Wolf. "Teichmuller space is not Gromov hyperbolic." Mathematical Sciences Research Institute (1994): preprint no. 011-94.

[33] Myasnikov, A. and A. Ushakov. "Random subgroups and analysis of the length-based and quotient attacks." Journal of Mathematical Cryptology 2, no. 1 (2008): 29-61.

[34] Rivin, I. "Walks on groups, counting reducible matrices, polynomials, and surface and free group automorphisms." Duke Mathematical Journal 142, no. 2 (2008): 353-79.

[35] Rivin, I. "Zariski density and genericity." International Mathematics Research Notices 2010, (2010): 3649-57.

[36] Sisto, A. "Contracting elements and random walks." (2011): preprint arxiv:1112.2666. 